\title{
High nonlinear figure of merit hydrogenated amorphous silicon optical fibers
}

\author{
L. Shen ${ }^{1}$, N. Healy ${ }^{1}$, P. Mehta ${ }^{1}$, T. D. Day ${ }^{2}$, J. V. Badding ${ }^{2}$, and A. C. Peacock ${ }^{1}$ \\ 1. Optoelectronics Research Centre, University of Southampton, Southampton SO17 1BJ, United Kingdom \\ 2. Department of Chemistry and Materials Research Institute, Pennsylvania State University 16802 PA, USA \\ ls1g11@orc.soton.ac.uk
}

\begin{abstract}
The wavelength dependence of the nonlinear properties of hydrogenated amorphous silicon core fibers are characterized across the two-photon absorption edge. These novel fibers exhibit a significant increase in nonlinear figure of merit around $2 \mu \mathrm{m}$.
\end{abstract}

(C) 2013 Optical Society of America

OCIS codes: $060.2270,060.2290,060.4370$.

\section{Introduction}

Hydrogenated amorphous silicon (a-Si:H) is becoming an increasingly popular material for nonlinear silicon photonics due to its low transmission losses, high Kerr nonlinearity and low fabrication costs [1]. Although the large bandgap of the amorphous material $(\sim 1.7 \mathrm{eV})$ suggests that nonlinear absorption should be modest at telecoms wavelengths which are past the two-photon absorption (TPA) edge, in practice the reported values of the TPA parameter $\beta_{\text {TPA }}$ are of a similar magnitude to crystalline silicon [2]. In this paper we extend the characterization of our a-Si:H core fibers beyond the telecommunications window of $\sim 1.5 \mu \mathrm{m}$ and present the first measurements of the transmission properties up to the edge of the mid-infrared regime $\sim 2-2.7 \mu \mathrm{m}$. The results show that the nonlinear figure of merit $\left(\mathrm{FOM}_{\mathrm{NL}}\right)$ increases dramatically over this region, with $\mathrm{FOM}_{\mathrm{NL}}>20$ around $2 \mu \mathrm{m}$ and above. The lower linear losses and negligible nonlinear losses that are measured past $1.7 \mu \mathrm{m}$ suggest that these fibers could find use for nonlinear applications in areas such as broadband and/or free-space communications, as well as some mid-infrared (mid-IR) gas sensing and medical applications.

\section{Theory and Characterization}

The propagation of optical pulses in silicon fibers can be described using the generalised nonlinear Schrödinger equation (NLSE) and the rate equation:

$$
\begin{gathered}
\frac{\partial A(z, t)}{\partial z}=-\frac{i \beta_{2}}{2} \frac{\partial^{2} A(z, t)}{\partial t^{2}}+i \gamma|A(z, t)|^{2} A(z, t)-\frac{1}{2}\left(\alpha_{l}+\sigma_{f}\right) A(z, t), \\
\frac{\partial N_{c}(z, t)}{\partial t}=\frac{\beta_{\mathrm{TPA}}}{2 h v_{0}} \frac{|A(z, t)|^{4}}{A_{\mathrm{eff}}^{2}}-\frac{N_{c}(z, t)}{\tau_{c}},
\end{gathered}
$$

where $A(z, t), \beta_{2}, \gamma, \alpha_{l}$, and $\sigma_{f}$ represent the slowly varying pulse envelope, the group velocity dispersion (GVD), nonlinear strength, linear loss, and the free carrier contribution, respectively. The nonlinear strength is complex and defined as, $\gamma=k_{0} n_{2} / A_{\text {eff }}+i \beta_{\mathrm{TPA}} / 2 A_{\text {eff }}$, where $n_{2}$ is the Kerr nonlinear coefficient, $\beta_{\mathrm{TPA}}$ is the TPA coefficient, and $A_{\mathrm{eff}}$ is the mode area. The free carrier contribution is described as $\sigma_{f}=\sigma(1+i \mu) N_{c}$, where $\sigma$ is the free-carrier absorption (FCA) coefficient, $\mu$ governs the free-carrier dispersion (FCD), and $\tau_{c}$ is the corresponding carrier life time.

Our silicon-core, silica-clad fibers are fabricated using the well-established high pressure microfluidic chemical technique [2]. The fiber used in our experiments was fabricated by depositing a-Si:H inside a $5.7 \mu \mathrm{m}$ silica capillary, and had a completely filled length of $2.4 \mathrm{~cm}$. Two laser sources: (i) a tunable $\mathrm{Cr}^{+}: \mathrm{ZnSe}$ laser and (ii) a Ti:sappire pumped optical parametric oscillator $(\mathrm{OPO})$ were used to characterize the linear and nonlinear transmission properties from $1.45-2.7 \mu \mathrm{m}$.

The linear losses were determined by the cutback technique and are plotted in Fig. 1(a). These results follow the same decreasing trend for increasing wavelength observed in our earlier measurements, and the losses in the range beyond $2 \mu \mathrm{m}$ are all less than $1 \mathrm{~dB} / \mathrm{cm}$. At the longest wavelength of $2.7 \mu \mathrm{m}$, the loss is $0.29 \mathrm{~dB} / \mathrm{cm}$, which is one of the lowest loss values recorded in this wavelength regime. Following this, we used the approach in Ref. [2] and measured the optical limiting data as a function of wavelength, from which we estimated the nonlinear TPA parameters $\beta_{\text {TPA }}$ as shown in Fig. 1(b). In these measurements a short piece fiber $(L=0.47 \mathrm{~cm})$ was used so that we could neglect 

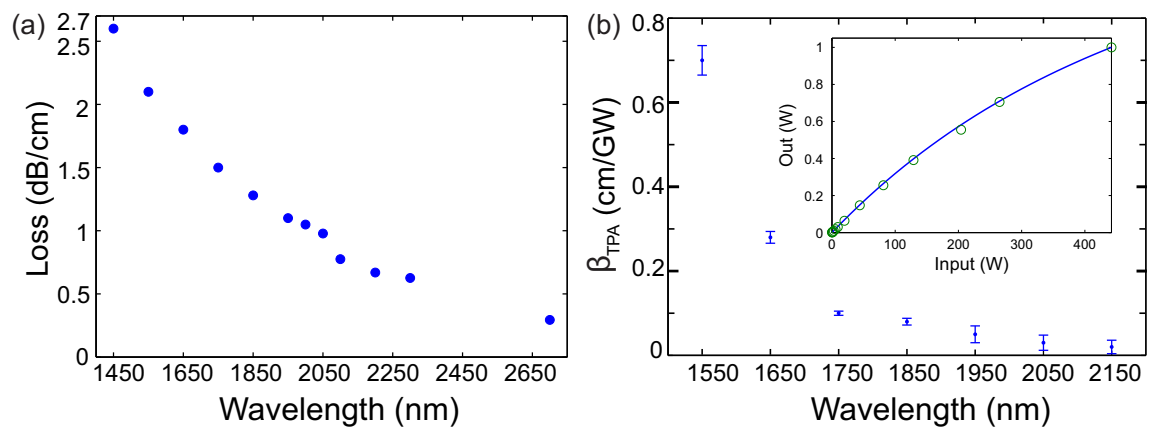

Fig. 1. (a) Linear loss measurements and (b) TPA parameter as functions of wavelength. Inset in (b), TPA fitting process at $1.55 \mu \mathrm{m}$. Error bars represent the uncertainty in the input powers.

the effects of dispersion, owing to $L$ being much shorter than the dispersion length for all wavelengths. We find that $\beta_{\text {TPA }}$ decreases rapidly over the wavelength range $1.5-1.65 \mu \mathrm{m}$, with $\beta_{\text {TPA }}=0.7 \pm 0.08 \mathrm{~cm} / \mathrm{GW}$ at $1.55 \mu \mathrm{m}$, before reaching a plateau at an essentially negligible value of $0.08 \mathrm{~cm} / \mathrm{GW}$ for wavelengths $>2 \mu \mathrm{m}$.

A series of self-phase modulation (SPM) experiments were then conducted to establish the Kerr nonlinear coefficient $n_{2}$, also as a function of wavelength. Here the size of the Kerr coefficient is obtained by fitting the spectral broadening of a short pulse propagating through the fiber with the solutions to Eq. (1) and Eq. (2), using the predetermined loss parameters. The corresponding values of $n_{2}$ are plotted in Fig. 2(a). The $n_{2}$ values first increase slightly up to $1.75 \times 10^{-13} \mathrm{~cm}^{2} / \mathrm{W}$ at $1.75 \mu \mathrm{m}$, and then drop to a modest value of $1.1 \times 10^{-13} \mathrm{~cm}^{2} / \mathrm{W}$ at $2.15 \mu \mathrm{m}$, which follows the predicated trend of the nonlinear Kramers-Krönig transformation [3]. Finally, we can combine the values for the TPA and Kerr parameters into a figure of merit $\mathrm{FOM}_{\mathrm{NL}}=\mathrm{n}_{2} / \lambda \beta_{\mathrm{TPA}}$, to investigate the wavelength dependence. As shown in Fig. 2(b), the calculated $\mathrm{FOM}_{\mathrm{NL}}$ of this a-Si:H core optical fiber increases monotonically towards longer wavelengths (up to $\sim 28$ ). We attribute this to the modest decrease of Kerr nonlinear coefficient $n_{2}$ and the dramatic drop in the $\beta_{\text {TPA }}$ value when moving across the TPA edge into the $2 \mu \mathrm{m}$ regime.
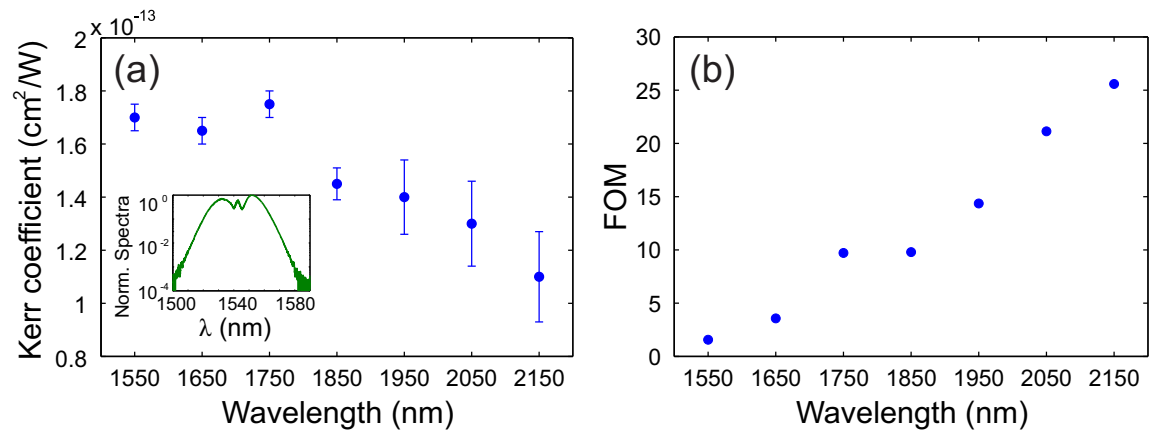

Fig. 2. (a) Wavelength dependence of the Kerr nonlinear coefficient $n_{2}$. Inset: Classic SPM spectra of a-Si:H core fiber centered at $1.54 \mu \mathrm{m}$. Error bars represent the uncertainty in the input powers.

(b) Wavelength dispersion of the nonlinear FOM.

\section{Conclusion}

We have characterized the transmission properties of hydrogenated amorphous silicon fibers extending from telecoms up to the edge of the mid-infrared, demonstrating some of the lowest losses in silicon waveguides in this wavelength region to date. The lower linear propagation losses and large $\mathrm{FOM}_{\mathrm{NL}}$ in these fibers at wavelengths $>2 \mu \mathrm{m}$ make them a suitable platform for applications for nonlinear optics.

\section{References}

1. B. Kuyken, H. Ji, S. Clemmen, S. K. Selvaraja, H. Hu, M. Pu, M. Galili, P. Jeppesen, G. Morthier, S. Massar, L.K. Oxenlwe, G. Roelkens, and R. Baets, "Nonlinear properties of and nonlinear processing in hydrogenated amorphous silicon waveguides," Opt. Express 19, B146-B153 (2011).

2. P. Mehta, N. Healy, N. F. Baril, P. J. A. Sazio, J. V. Badding, and A. C. Peacock, "Nonlinear transmission properties of hydrogenated amorphous silicon core optical fibers," Opt. Express 16, 16826-16831 (2010).

3. A. D. Bristow, N. Rotenberg, and H. M. Van Driel, "Two-photon absorption and Kerr coefficients of silicon for 850-2200 nm,” Appl. Phys. Lett. 90, 191104 (2007). 